\title{
Intricate interpretation of etiology-specific outcome comparison in patients with hepatocellular carcinoma
}

\author{
Hwi Young Kim ${ }^{1}$ and Jean-François Dufour ${ }^{2}$ \\ 'Department of Internal Medicine, College of Medicine, Ewha Womans University, Seoul, Korea; ${ }^{2}$ Hepatology, Department of Clinical \\ Research, University of Bern, Bern, Switzerland
}

\section{Dear Editor,}

We have read Kumar et al.'s study ${ }^{1}$ published in the previous issue of Clinical and Molecular Hepatology with great interest, which compared the characteristics and outcomes in patients with nonalcoholic steatohepatitis (NASH)-associated hepatocellular carcinoma (HCC) versus those with alcoholic steatohepatitis (ASH)-associated HCC. The study reported several differences in characteristics, such as age, sex, and comorbidities, between the two groups, whereas their survival rates as well as liver- and tumor-related factors were comparable. Although the current study was a retrospective analysis of a single-center cohort, it still offers a number of relevant points for further consideration.

First, patients with nonalcoholic fatty liver disease (NAFLD) or alcohol-related liver disease (ALD) are prone to deficient surveillance. ${ }^{2,3}$ Patients with undiagnosed alcohol-related cirrhosis also contribute to the low rate of surveillance as well as late diagnosis of HCC, due to their low compliance with regular appointments. ${ }^{4}$ $\mathrm{NASH}$-related cirrhosis is a well-known indication of surveillance for HCC. However, for patients with non-cirrhotic NASH (or even non-NASH NAFLD), there has been no consensus on whether to offer surveillance or not. In Kumar et al.'s study, ${ }^{1}$ the frequencies of non-cirrhotic patients with NASH-HCC and ASH-HCC were similar, whereas significantly more patients with ASH-HCC were diag- nosed with surveillance compared to those with NASH-HCC. The difference in surveillance possibly resulted from the small sample size, considering the low rate of surveillance and late diagnosis in both etiology groups. ${ }^{2,3}$ Older age in patients with NASH-HCC may result from the lower surveillance rate, as discussed in Kumar et al.'s study.' Moreover, delayed diagnosis of HCC can lead to large tumor and poor outcomes in patients with NAFLD or ALD. Appropriate surveillance strategy for patients with NAFLD or ALD is urgently needed in order to discover more patients at earlier stages, when they are amenable to curative therapies. However, non-cirrhotic patients existed in both groups of Kumar et al.'s study, ${ }^{1}$ adding difficulty in selecting the target population for surveillance.

Second, the presence of modifiable lifestyle factors and their impact on the outcome should be studied. In particular, there might have been NASH-HCC patients with nonsignificant (<60 g/day) alcohol consumption in Kumar et al.'s study, ${ }^{1}$ which otherwise could be defined as significant, if the amount exceeded $>21$ (14) standard drinks/week in men (women). ${ }^{5}$ The exact proportion of NASH-HCC patients with alcohol consumption amount between 21 (14) standard drinks/week and $<60 \mathrm{~g} /$ day is not assessable, due to the lack of information. Likewise, obese ASH$\mathrm{HCC}$ patients (body mass index, $\geq 25 \mathrm{~kg} / \mathrm{m}^{2}$ ) might also exist in

\begin{abstract}
Abbreviations:
ALD, alcohol-related liver disease; ASH, alcoholic steatohepatitis; HCC, hepatocellular carcinoma; HCV, hepatitis C virus; NAFLD, nonalcoholic fatty liver disease; NASH, nonalcoholic steatohepatitis
\end{abstract}

\section{Corresponding author : Jean-François Dufour}

Hepatology, Department of Clinical Research, University Clinic for Visceral Surgery and Medicine, University of Bern, D115, Inselspital, Bern 3010, Switzerland

Tel: +41 3163280 26, Fax: +41 316327489

E-mail: jean-francois.dufour@dbmr.unibe.ch

https://orcid.org/0000-0002-8062-1346 
the study population. A recent study from a prospective European cohort demonstrated that smoking was an independent predictor of survival in hepatitis B or C-infected HCC patients. ${ }^{6}$ In addition, NASH-HCC patients suffer from various comorbidities, such as cardiovascular diseases. ${ }^{7}$ Therefore, multiple coexistence of lifestyle risk factors or their comorbid conditions may differ at individual level, and this needs to be verified from the preventive perspective.

Third, the interpretation of etiology-specific outcomes and their comparison requires caution, possibly due to selection bias. Previous studies reported controversial results, especially in terms of survival in NAFLD- or NASH-HCC patients. A prospective multicenter observational study from Italy reported similar survival rates between NAFLD-HCC and hepatitis C virus (HCV)-related HCC groups after patient matching, where the difference in mortality was driven by cardiovascular causes. ${ }^{8}$ Another Italian study showed negative effect of alcoholic etiology on survival compared to HCV-HCC, which disappeared after adjustment for confounding factors and stratification by tumor stage. ${ }^{2}$ In cohort studies to evaluate and compare etiology-specific outcomes, efforts are needed to adjust the differences in baseline characteristics (e.g., using a propensity score analysis) and to minimize lead-time bias, considering the imbalance in surveillance rates between groups.

In summary, Kumar et al.'s study' provided intriguing yet difficult aspects of HCC management, with its epidemiology changing over the last decade. Viral etiologies that underscore the importance of lifestyle-related HCC, such as ALD or NAFLD-HCC, are anticipated to be controlled in the near future. Appropriate surveillance strategy and early diagnosis based on larger-scale, welldesigned prospective studies may be the most challenging tasks for improving the outcome.

\section{Authors' contribution}

Conception, manuscript writing and critical revision: HYK and J-FD

\section{Conflicts of Interest}

The authors have no conflicts to disclose.

\section{References}

1. Kumar R, Goh BG, Kam JW, Chang PE, Tan CK. Comparisons between non-alcoholic steatohepatitis and alcohol-related hepatocelIular carcinoma. Clin Mol Hepatol 2020;26:196-208.

2. Bucci L, Garuti F, Camelli V, Lenzi B, Farinati F, Giannini EG, et al. Comparison between alcohol- and hepatitis $C$ virus-related hepatocellular carcinoma: clinical presentation, treatment and outcome. Aliment Pharmacol Ther 2016;43:385-399.

3. Singal AG, Yopp AC, Gupta S, Skinner CS, Halm EA, Okolo E, et al. Failure rates in the hepatocellular carcinoma surveillance process. Cancer Prev Res (Phila) 2012;5:1124-1130.

4. Ganne-Carrié N, Nahon P. Hepatocellular carcinoma in the setting of alcohol-related liver disease. J Hepatol 2019;70:284-293.

5. Chalasani N, Younossi Z, Lavine JE, Charlton M, Cusi K, Rinella M, et al. The diagnosis and management of nonalcoholic fatty liver disease: practice guidance from the American Association for the Study of Liver Diseases. Hepatology 2018;67:328-357.

6. Kolly P, Knöpfli M, Dufour JF. Effect of smoking on survival of patients with hepatocellular carcinoma. Liver Int 2017;37:1682-1687.

7. Degasperi E, Colombo M. Distinctive features of hepatocellular carcinoma in non-alcoholic fatty liver disease. Lancet Gastroenterol Hepatol 2016;1:156-164.

8. Piscaglia F, Svegliati-Baroni G, Barchetti A, Pecorelli A, Marinelli $S$, Tiribelli $C$, et al. Clinical patterns of hepatocellular carcinoma in nonalcoholic fatty liver disease: a multicenter prospective study. Hepatology 2016;63:827-838. 